\title{
Estrategia de mejora para el aumento de la competitividad de los productos - servicios hoteleros de ciudad del grupo de turismo Gaviota s.a.
}

\section{Improvement strategy to increase the competitiveness of products - city hotel services of the Gaviota tourism group s.a.}

Ms.C. Mitsue Eumary Spengler Betancourt. ${ }^{1}$ \& Ms.C. Jimmy Eduardo Ascón Villa. ${ }^{2}$

\begin{abstract}
The research addresses competitiveness in city hotels from the comparison between the indicators of the main tourism groups at the national level: Gran Caribe S.A, Cubanacán S.A and Gaviota S.A. In this case, the Gaviota Group is privileged for being the one with the highest housing growth, perspectives in the development of tourism based on compliance with the Party's guidelines and national strategic projection. To demonstrate the current situation of city products-services in the Gaviota Group, the sample of three hotels located in the Miramar area in the Havana destination is analyzed: Hotel Memories Miramar, Hotel H10 Habana Panorama and Hotel Four Points By Sheraton La Havana.

In order to achieve the stated purpose, various bibliographic sources were consulted, selecting the tools "Analysis of the five competitive forces" and "Analysis of the value chain" for the assessment of Grupo Gaviota S.A. In the analysis of the position of the Gaviota Group in the hotel sector focused on the city modality, the Delphi Method was used. In addition, an analysis was carried out of the competitive advantages that Gaviota S.A. has operating under the form of administration: Management Contract. Interviews were conducted with top-level managers in the selected hotels to determine the elements that add value to the chain. Among the main results are the Key Success Factors,

\footnotetext{
${ }^{1}$ Universidad Estatal Amazónica, Centro de Posgrado, Maestrante en Turismo, Mención Gestión del Turismo, Puyo-Ecuador, xas337@gmail.com

${ }^{2}$ Universidad Estatal Amazónica, Facultad Ciencias de la Vida, Carrera de Turismo, Puyo-Ecuador, luisauquilla10@gmail.com
} 
determined by the Distinctive Capabilities and Competitive Advantages, as well as the possible strategies to increase its competitive situation.

Key Words: Competitiveness, City Hotels, Key Success Factors And Strategies.

\section{Resumen}

La investigación aborda la competitividad en los hoteles de ciudad a partir de la comparación entre los indicadores de los principales grupos de turismo a nivel nacional: Gran Caribe S.A, Cubanacán S.A y Gaviota S.A. En este caso se privilegia el Grupo Gaviota por ser el de mayor crecimiento habitacional, perspectivas en el desarrollo del turismo a partir del cumplimiento de los lineamientos del Partido y proyección estratégica nacional. Para demostrar la situación actual de los productos-servicios de ciudad en el Grupo Gaviota se analiza la muestra de tres hoteles ubicados en la zona de Miramar en el destino La Habana: Hotel Memories Miramar, Hotel H10 Habana Panorama y Hotel Four Points By Sheraton La Habana.

En aras de lograr el propósito trazado se consultaron diversas fuentes bibliográficas, seleccionándose las herramientas "Análisis de las cinco fuerzas competitivas” y "Análisis de la cadena de Valor" para la valoración del Grupo Gaviota S.A. En el análisis de la posición del Grupo Gaviota en el sector hotelero enfocado a la modalidad de ciudad, se empleó el Método Delphi. Además se realizó un análisis de las ventajas competitivas que posee Gaviota S.A operando bajo la forma de administración: Contrato de Gestión. Se realizaron entrevistas a los mandos de primer nivel en los hoteles seleccionados para determinar los elementos que aportan valor a la cadena. Entre los principales resultados se encuentran los Factores Claves de Éxito, determinados por las Capacidades Distintivas y las Ventajas Competitivas, así como las posibles estrategias para aumentar su situación competitiva.

Palabras Clave: Competitividad, Hoteles de ciudad, Factores claves de éxito y Estrategias.

\section{Introducción}

El cambio es un elementos que condiciona en la actualidad la gestión empresarial, como parte de un mundo globalizado en el que aumentan las facilidades de acceso a la información y al conocimiento, pero de manera condicionada por factores negativos relacionados a la crisis económica internacional desarrollada hace ya varios años a nivel internacional, se hace necesario el estudio de los elementos que determinan la capacidad para el desarrollo en los destinos turísticos. Es por ello que el Caribe viendo afectado notablemente la competitividad de sus mercados se proyecta en nuevas estrategias que le permitan la integración al entorno, y el aprovechamiento de sus ventajas.

El cambio es un elementos que condiciona en la actualidad la gestión empresarial, como parte de un mundo globalizado en el que aumentan las facilidades de acceso a la información y al conocimiento, pero de manera condicionada por factores negativos relacionados a la crisis económica internacional desarrollada hace ya varios años a nivel internacional, se hace necesario el estudio de los elementos que determinan la capacidad 
para el desarrollo en los destinos turísticos. Es por ello que el Caribe viendo afectado notablemente la competitividad de sus mercados se proyecta en nuevas estrategias que le permitan la integración al entorno, y el aprovechamiento de sus ventajas.

El aumento de la competencia ha estado determinado en el área, fundamentalmente en las principales modalidades de Sol y Playa y el Turismo de Ciudad, intensificando la rivalidad entre cadenas hoteleras orientadas a los principales mercados que visitan la región. La situación se agudiza debido a que los grupos de turismo presentes en los principales destinos cubanos trabajan con el mercado canadiense y europeo, ofreciendo facilidades similares para el desarrollo de los productos-servicios hoteleros en los destinos de ciudad.

El entorno descrito demanda una constante transformación en la gestión de las empresas, principalmente en el alojamiento enfocado a dichas modalidades, para que la administración de las mismas garantice una serie de estándares que mediante la planificación, organización, gestión y control de los recursos disponibles permita cumplir los objetivos con eficacia y eficiencia, aumentando la competitividad en el mercado a partir del diseño estratégico empresarial.

La competitividad se presenta por el economista Porter (1990), como capacidad de una organización de crear ventajas competitivas sostenidas frente a sus rivales en un entorno de elevada competencia, obteniendo mayor rentabilidad y creciendo más que estos al conquistar más consumidores e incluso arrebatarles su propia clientela ofreciéndole un mayor valor.

Relacionado a los elementos contenidos en el alcance de su concepto debe perfilarse la importancia del tema dado el objetivo permanente del Grupo de Turismo Gaviota S.A interesado en realizar estudios para mejorar de forma continua su gestión y alcanzar la excelencia en un estado cuantitativa y cualitativamente superior.

Para un análisis más objetivo de la competitividad de la empresa Porter (1990) propone dos modelos importantes: el Modelo de las Cinco Fuerzas Competitivas y el Análisis de la Cadena de Valor.

Gárciga, (1999) y por Porter, (1985), indican claramente que dentro de los factores que determinan la competitividad y los que permiten marcar ventajas competitivas, existen agentes internos y externos, que se traducen en la cadena de valor de la empresa u organización.

La cadena valor desarrollada por Porter (1988), constituye uno de los instrumentos más ricos y populares para el análisis y diagnóstico interno de la empresa. El objetivo de la cadena de valor consiste en identificar las fuentes internas de ventajas competitivas, intentando comprender cómo se va generando el valor en la organización.

Es necesario analizar el sector en el que se desenvuelve la empresa, a través de las cinco fuerzas competitivas que actúan en él y su relación con los factores claves del éxito en el mismo, para el aumento de la competitividad. 
Según Eccles (1993), son condiciones internas o externas claves para que la estrategia de la empresa sea exitosa. Por ejemplo: aceptación de usuarios, movimientos de los competidores, recursos humanos o financieros, o como asegura este autor, eventos, condiciones, circunstancias o actividades en las cuales resultados satisfactorios asegurarán un desempeño competitivo para la organización. Son elementos cruciales para el éxito de una empresa durante el horizonte de la planeación.

Para los autores Jonson y Acholes (2002), los FCE son aquellas características del producto que son altamente valorados por un grupo de clientes y en las que, por lo tanto, la organización debe de tener éxito para superar a los competidores. Este es el criterio con el que se trabaja en la presente investigación.

Los factores claves del éxito (FCE) son aquellos elementos sobre los que se basa la competencia en el mercado. De acuerdo a dichos factores la empresa debe desarrollar sus capacidades distintivas y lograr ventajas competitivas. (Ronda, 2015)

Siguiendo esta noción, para Gárciga (1999), existen 5 puntos clave para analizar los FCE.

- $\quad$ La posición en el mercado.

- La posición de la empresa en materia de costes de suministro, producción y comercialización.

- La imagen y la implantación comercial.

- Las competencias técnicas y el dominio tecnológico.

- La rentabilidad y el poder económico.

Para lograr ventajas competitivas en relación a los contrincantes en un determinado sector es esencial dominar los factores claves del éxito mejor que ellos y para esto la empresa tendrá que contar con un grupo de capacidades.

El presente estudio se orienta el análisis de la competitividad en el Grupo de Turismo Gaviota S.A. Tomando en cuenta el aumento de la situación competitiva en el destino La Habana y más particularmente en la zona de Miramar, se hace necesario el estudio de los factores claves del éxito en el Grupo de Turismo Gaviota S.A. dada la variedad de hoteles de prestigio que son gestionados por otros grupos de turismo de probado éxito en Cuba como lo son Cubanacán y Gran Caribe, que además emplean formas de gestión hotelera como la empresa mixta, contrato de gestión o la administración propia; aunado a que en la zona como en el resto de la capital, destacan alojamientos y casas de renta del sector no estatal que ofrecen servicios de alta relación calidad-precio a los clientes, determinando así un aumento en los alojamientos turísticos, y una variante más económica para los clientes que sólo se diferencia en la facilidad de salones para reuniones y otros servicios más especializados por parte de los hoteles de la propuesta estatal.

El aumento de la oferta para los clientes hace necesario el estudio de los factores claves del éxito para los grupos hoteleros, y la atenta selección de proveedores, así como el conocimiento de la situación competitiva en el entorno, y las diferencias con el resto de 
los grupos de turismo para con base en sus ventajas y desventajas competitivas proponer nuevas estrategias.

La Dirección Estratégica Integrada es un enfoque cualitativamente superior de la dirección estratégica que se expresa en la coordinación de todo el sistema de relaciones internas y externas, con sentido de cooperación y plena participación, para alcanzar la visión de la organización, y producir resultados sinérgicos de alto impacto económico y social, en el presente y en el futuro en el cumplimiento de su objeto social. Está basada en el liderazgo de las relaciones en toda la organización; actúa sobre las relaciones de carácter interno y externo, actuales y futuras y está enfocada a romper todas las barreras que limitan la toma de decisiones efectivas en función del cumplimiento de la visión. La Dirección Estratégica Integrada incorpora una nueva cualidad al sistema de dirección empresarial, convirtiéndola en una alternativa de solución a la contradicción que enfrentan las empresas, que operan en entornos complejos, las que de un lado requieren de alta autonomía de todos sus eslabones para dar respuesta ágil, y de otro lado, requieren que sus eslabones trabajen en sistema, como un todo, para alcanzar los objetivos de la empresa. (Ronda, 2016)

Tomando en cuenta el criterio de Wootton y Horne (2012) pensar estratégicamente va mucho más allá que planificar o dirigir por estrategias. El pensamiento estratégico implica el desarrollo de una serie de habilidades que los directivos requieren en el mundo de hoy, donde las diferentes variables del entorno y su comportamiento le imprimen una alta complejidad a los fenómenos empresariales. El pensamiento estratégico implica tres actividades esenciales: obtener información, formular ideas y planear acciones.

El Objetivo general de la investigación es elaborar una propuesta de estrategias para la mejora de la competitividad de los productos-servicios hoteleros del Grupo de turismo Gaviota S.A.

$\mathrm{Su}$ importancia radica en que en la realidad cubana actual existe una problemática determinada por la interrelación entre los estándares de las cadenas, los segmentos de mercado a los que se orienta su gestión y la modalidad turística asociada al alojamiento, lo que se encuentra influenciado por la contratación extranjera de cadenas especializadas en el turismo convencional para el desarrollo de hoteles de ciudad u otra modalidad en los que no presenta la misma experiencia, y por tanto se hace necesario el estudio de debilidades asociadas a su competitividad, y en relación a otras cadenas o grupos nacionales representativos dentro del sistema de turismo nacional.

Para el trabajo del Grupo de Turismo Gaviota S.A. y la gestión de los hoteles que se tomaron como objeto de estudio, es necesaria porque integra técnicas comúnmente empleadas en análisis aislados y las complementa con un análisis Delphi para el logro del análisis de la atractividad del mercado y su posición competitiva en aras de lograr un estudio global de la situación del grupo en su entorno. El estudio resulta novedoso, siendo la primera vez que se desarrolla un análisis holístico sobre los factores claves para el éxito de sus hoteles de ciudad en el destino La Habana y con respecto a otros grupos de turismo, lo que permite conocer la realidad sobre su situación competitiva y en consecuencia 
proponer estrategias y acciones para fortalecer sus capacidades distintivas y generar nuevas ventajas competitivas.

\section{Desarrollo}

\section{Resultados del modelo análisis de las cinco fuerzas competitivas}

Tomando en consideración que la gestión de hoteles de ciudad representa para los resultados financieros de Gaviota al menos el 32\% de sus habitaciones, se enfatizó en el estudio de las ventajas competitivas que posee el Grupo y de cuáles deberá desarrollar para mejorar su posición en el sector hotelero enfocado a la modalidad de ciudad.

Con dicho propósito se evaluó el sector hotelero enfocado a la modalidad de ciudad en Cuba a través del Modelo de las Cinco Fuerzas Competitivas de Michael Porter, llegando a los siguientes resultados:

- El poder negociador de los proveedores es favorable para la industria hotelera de ciudad cubana. Los proveedores se encuentran concentrados y el hotelero no posee mayor poder negociador (los proveedores son fijados de manera centralizada, por lo que no favorecen en precios y condiciones de pago). En Cuba se observa una tendencia a la subida de precios lo que limita el poder negociador de los hoteleros pues muchas veces sólo pueden comprar a un proveedor para una calidad y cantidad determinada.

- La fuerza poder negociador de los clientes es alta para el sector de ciudad porque un pequeño grupo de TT.OO. concentra el mayor número de turistas de ciudad, lo que determina su dependencia en el volumen de compra.

- En tales escenarios, los hoteleros de ciudad presentan una alta rivalidad frente a un cliente que presenta amplias facilidades para asumir nuevos proveedor de sus productos-servicios de ciudad.

- La amenaza de nuevos entrantes no presenta elevadas barreras de entrada, ya que la política del país privilegia los logros alcanzados en la gestión del Grupo de Turismo Gaviota, por lo que el sector no es de fácil acceso para los nuevos competidores determinando la posición competitiva satisfactoria del grupo.

- En Cuba no existe para el grupo una alta disponibilidad de productos sustitutos, mientras que en el Caribe estos presentan un rango medio mayor. Es por ello que la fuerza amenaza de nuevos sustitutos no clasifica como hostil para el sector de ciudad, aunque existe una competencia que debe ser estudiada.

\section{Resultados del modelo análisis de la matriz del perfil competitivo.}

Se puede concluir que existe una fuerte rivalidad competitiva entre las empresas del sector hotelero de ciudad, pues las condiciones existentes intensifican cada una de las cinco fuerzas. Dichos resultados están reflejados en las Matrices de Perfil Competitivo que se exponen a continuación: 
Tabla 1. Matriz del Perfil Competitivo para el sector hotelero de ciudad en Cuba (adaptado de Porter):

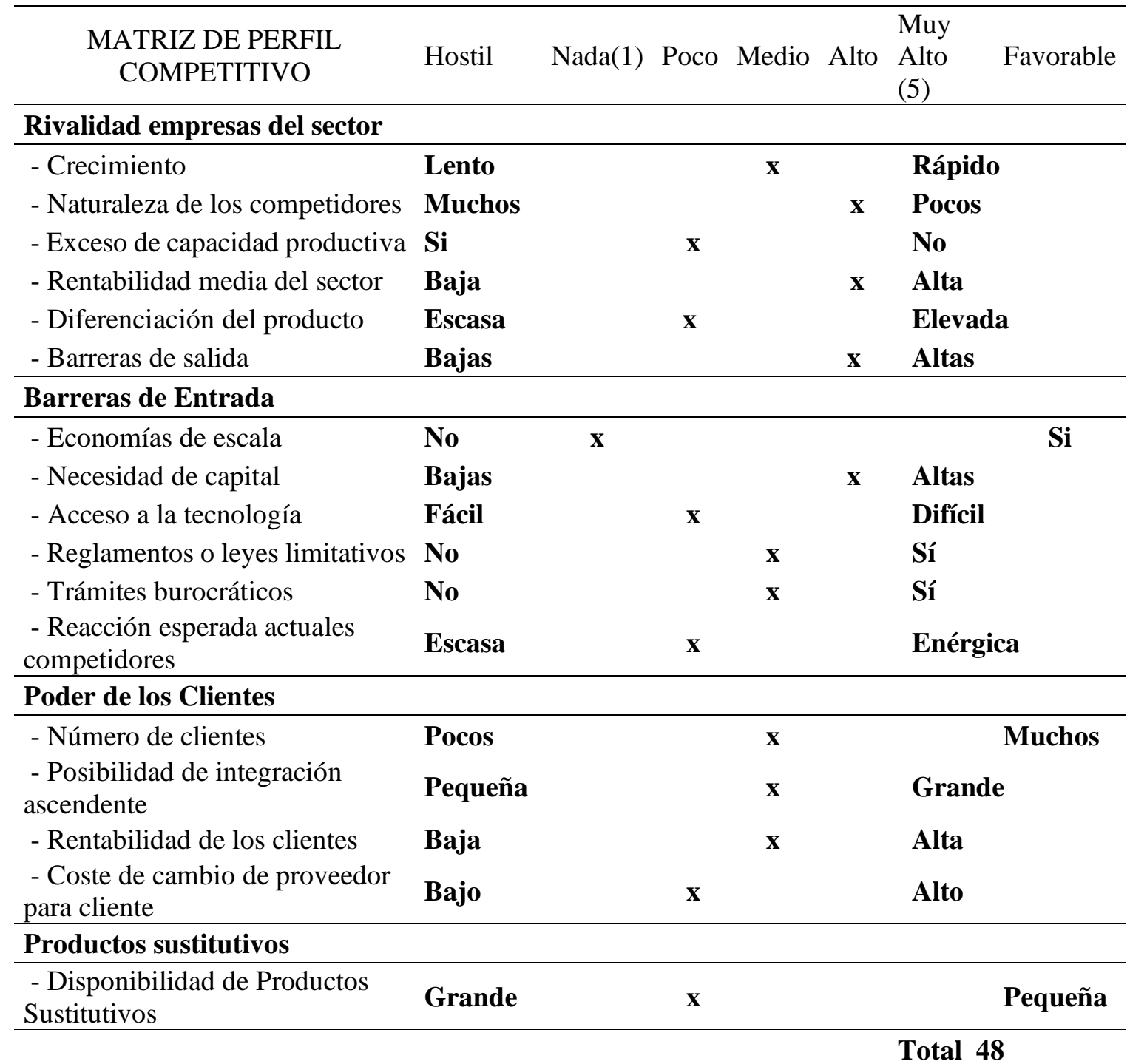

Indicando que:

El sector hotelero enfocado a la modalidad turismo de ciudad en Cuba, se caracteriza por una alta rivalidad competitiva, a partir de una situación favorable de los mercados para el desarrollo de los competidores del Grupo Gaviota, pero sus oportunidades se ven limitadas para el logro de un posicionamiento competitivo superior.

Para las empresas hoteleras de ciudad en el destino país Cuba las barreras de salida son muy altas ya que se puede cambiar de sector o mover el capital a otro tipo de negocio solamente con la autorización del estado.

La poca diferenciación entre los productos-servicios de ciudad incita a que los turoperadores y agencias de viaje no presenten dificultades para cambiar de proveedor, y que la posibilidad de integración ascendente en la cadena de valor, para los hoteleros 
cubanos sea media en función de sus capacidades para la ejecución de nuevas formas estratégicas de gestión con marcas de prestigio internacional, lo que presupone una fuente para el desarrollo de ventajas competitivas en tal sentido, asociadas a las ventajas que estas ofrecen.

Gaviota se ha orientado como grupo en el desarrollo de contratos de gestión que permiten obtener la experiencia de marcas prestigiosas que han aportado en cuanto a estándares y know how lo que responde a las necesidades de la empresa en el sector turístico cubano.

Respecto a sus productos-servicios las modificaciones podrían consistir en incluir servicios que faciliten el valor añadido ampliando los estándares en adecuación a los segmentos de mercado reales con que trabajan los grupos para elevar los niveles de satisfacción con la calidad y aumentar la rentabilidad.

Por otro lado, la política comercial debe orientarse a la búsqueda de mejores relaciones contractuales con los intermediarios.

\section{Análisis del dominio de los factores claves de éxito en el sector hotelero de ciudad de la habana para el grupo Gaviota s.a.}

Los resultados del cuestionario aplicado a expertos evidencian que por lo general el grado de dominio de Gaviota es inferior al de la mayoría de sus competidores desde el análisis de las marcas extranjeras con presencia en Cuba y superior con respecto a los grupos cubanos Gran Caribe y Cubanacán en casi la totalidad de los factores. Los resultados obtenidos se presentan en el siguiente gráfico:

La empresa presenta mejor posición que Gran Caribe y Cubanacán en 14 de los 16 factores, y estas cadenas sólo predominan ante el Grupo en 2 FCE respectivamente, como refleja el siguiente gráfico:

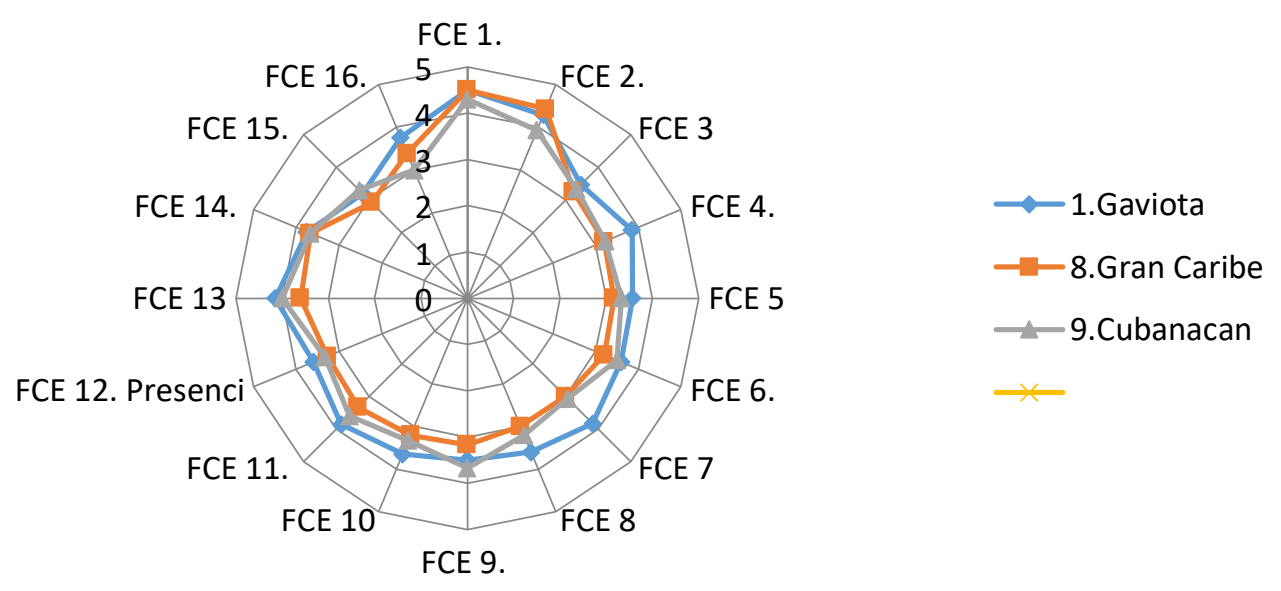

Figura 1. Comparación de los FCE entre los grupos hoteleros cubanos

Fuente: Elaboración propia. 
Los 9 primeros factores enunciados se encuentran estrechamente vinculados al Producto. Comparando a las 3 empresas cubanas desde esta perspectiva se observa que prevalece un grado de dominio aceptable, y que las diferencias entre ellas, como promedio, son mínimas.

Los FCE 10, 11 y 12 se corresponden con la Promoción, respecto a esta todos los FCE asociados se hallan en el nivel de dominio aceptable y las diferencias entre las empresas son mínimas.

Los FCE 13, 14, 15 y 16 se relacionan con la Comercialización, donde Gaviota no domina la mitad de los FCE asociados y la otra mitad se halla en el rango de dominio aceptable, aunque se perciben similares resultados.

A continuación, se agrupan los resultados anteriores en las áreas claves en que se enmarcan los FCE del sector con vistas a determinar la situación del Grupo respecto a cada una de ellas.

Como se aprecia en dicha tabla, los 16 FCE identificados integran las áreas "Posición en el Mercado" e "Imagen e Implantación Comercial." De ellos el Grupo domina en un grado relativamente alto 3 y en el rango mínimo aceptable 13; obteniendo como promedio una puntuación de 3.76, con la que se ubica en la ante antepenúltima posición.

En las áreas "Posición de la empresa en materia de costes de suministro, producción y comercialización" y "Rentabilidad y poder económico" compuestas por los FCE 14 y 16, Gaviota se coloca en el antepenúltimo y ante antepenúltimo lugares respectivamente.

En el área "Competencias técnicas y dominio tecnológico" formada por los FCE 12, 13 Gaviota ocupa el ante antepenúltimo lugar y en el 14 y 15 ocupa nuevamente el ante penúltimo lugar.

De lo que se concluye que:

Dentro de las 5 áreas claves Gaviota en 3 se halla en la ante antepenúltima posición y en 2 en la ante penúltima antecediendo a Gran Caribe y Cubanacán, excepto en el FCE 15. Es decir, que no domina ninguno de los FCE en un grado tal que pueda afirmarse que presenta ventajas competitivas respecto a sus rivales en las 5 áreas claves para analizar los factores claves de éxito ya que, como se ha venido reiterando, una empresa ha ganado una ventaja competitiva cuando domina un FCE mejor que la competencia, lo que no se cumple para el caso del Grupo Gaviota.

La organización aún debe, partiendo de sus recursos físicos, humanos, tecnológicos etc. (o buscando los necesarios) desarrollar conocimientos y habilidades para competir que no sean de fácil reproducción por sus competidores y que expresen su comprensión del funcionamiento del sector. Estas condiciones corroboran la situación desfavorable dentro de un sector de elevada rivalidad competitiva. 
Capacidades distintivas del grupo gaviota s.a. y su relación con los factores claves de éxito del sector hotelero.

Se determinó la correspondencia entre los factores claves de éxito reconocidos para el sector y las capacidades distintivas que identifican a la empresa. La correlación entre factores y capacidades sería necesaria para garantizar la posibilidad de ventajas competitivas para el Grupo en la modalidad que analizamos, de no darse, será preciso que la organización asegure los recursos para desarrollar las potencialidades indispensables.

Las Capacidades Distintivas del Grupo Gaviota identificadas según criterio de expertos son:

1. Experiencia de más de 20 años en la explotación de hoteles de ciudad.

2. Alto nivel cognoscitivo de los mercados de turismo de ciudad.

3. Asociación con cadenas hoteleras extranjeras de reconocido prestigio internacional en la gestión de alojamientos turísticos en las ciudades.

4. Más del $88 \%$ de las políticas de las marcas implementadas en la gestión de sus hoteles de ciudad, que solo se ven afectadas por los estándares tecnológicos.

5. Manual de procedimientos en el $92 \%$ de los hoteles de ciudad.

6. Diseñadas las actividades de animación pasiva.

1. El estudio arrojó que el Grupo Gaviota debiera poseer, además, las siguientes Capacidades Distintivas:

2. Implementación de Sistemas de Gestión de la Calidad en todos sus hoteles de ciudad.

3. Aplicación de la Gestión por Procesos en todos los hoteles ciudad.

4. Estudio de oportunidad de proyectos para activos actuales y futuras inversiones.

Diseño estratégico propuesto para el grupo Gaviota s.a. en función de los fce analizados, con base en los resultados.

Tabla 2. Relación entre FCE, Estrategias asociadas a las Capacidades Distintivas y posibles acciones generadoras.

\begin{tabular}{|c|c|c|}
\hline FCE & $\begin{array}{l}\text { ESTRATEGIA } \\
\text { ASOCIADAS A LAS } \\
\text { CAPACIDADES } \\
\text { DISTINTIVAS }\end{array}$ & ACCIONES GENERADORAS \\
\hline $\begin{array}{l}\text { 1. Calidad del } \\
\text { inmueble. }\end{array}$ & $\begin{array}{lr}\text { Estudio de } & \text { de } \\
\text { oportunidad del } \\
\text { negocio para } \\
\text { activos actuales y } \\
\text { próximas } \\
\text { inversiones. }\end{array}$ & $\begin{array}{l}\text { Evaluar las inversiones futuras para las } \\
\text { instalaciones en categoría de proyecto para } \\
\text { invertir en ellas o pasarlas a cadenas que } \\
\text { reúnan los requisitos que demandan los } \\
\text { segmentos de mercado. }\end{array}$ \\
\hline
\end{tabular}




2. Cercanía a
aeropuertos y
centro de la
ciudad.

3. Planta hotelera según estándares internacionales.

\section{Estudio de \\ oportunidad del}

negocio para Evaluar las instalaciones antes de invertir en activos actuales y las proyecciones futuras y establecer próximas contrato de gestión con las cadenas que inversiones reúnan las características en cuanto a incluidas en la estándares de la marca. proyección del grupo.

Asociación con cadenas hoteleras extranjeras de Continuar invirtiendo en el crecimiento de la reconocido prestigio internacional.

planta hotelera de alto estándar del grupo.
Optimizar la planta hotelera actual.

Aplicar ingeniería Aumentar el estándar de restaurantes de menú a los especializados y temáticos. Realizar nuevas 4. Oferta puntos de venta propuestas de ofertas típicas de la cocina gastronómica gastronómica para cubana. Impartir cursos asociados a la variada aumentar la actividad de cantina y sommeliería. atractividad de las ofertas

Diseñar y evaluar cartas menús por temporada.

5. Animación Diseño de Efectuar eventos nacionales e pasiva actividades de internacionales de para fomentar la animación pasiva creatividad y el conocimiento en diferentes temáticas de interés para los segmentos de los hoteles. Transmitir música grabada en frecuencia baja o de relax en la piscina, grupo musical tradicional cubano en el lobby y restaurantes. Exposiciones de arte. Pinturas cubanas en las áreas de los hoteles.

6. Calidad del 1. Implementar Actualizar los estándares de las marcas servicio Gestión de la productos-servicios hoteleros de ciudad. Calidad

2. Implementar Diseñar sistemas de retroalimentación y la Gestión por compensación de clientes.

Procesos en todos los hoteles.

Categorizar las instalaciones hoteleras.

Actualizar los manuales de operación por marca. 
Lograr una mejor composición funcional y presentación de la papelería informativa en las habitaciones.

Actualizar el Manual de Operaciones del Grupo Gaviota S.A.

7. Producto Empleo de las Realizar al menos un taller trimestral de consistente previsiones en el diseño de nuevos productos y servicios a control de costos y partir de los estándares de los proveedores. gastos con los proveedores para el diseño de nuevos productosservicios.

8. Realizar nuevos Desarrollar nuevos producto de ciudad con Diferenciación estudios de la valores añadidos como inclusión en y valores demanda y la excursiones de la zona, segmentos que añadidos competencia para buscan bienestar y desarrollo de actividades determinar qué cognitivas como talleres, eventos, concursos, servicios de valor entre otras. añadido incluir.

9. Imagen 1. Experiencia Adecuar los estándares de las cadenas y su propia por de casi 15 años en know how no solo para los segmentos marcas la explotación de habituales de dichas cadenas sino también hoteles de ciudad para los segmentos metas para los cuales 2. Alto nivel fueron concebidos en su categoría de cognoscitivo de los proyecto los hoteles velando por el mercados de mantenimiento de la correspondencia ciudad calidad y precio.

3. Política de marcas aplicada a los productosservicios hoteleros de ciudad.

10. Calidad de Diseño de sueltos y Apoyar el plan de comercialización los soportes papeletica ampliando en número y calidad las promocionales promociones en formato impreso y digital.

11. Presencia Actualización de Implementar acciones de Co- Marketing con de marcas en catálogos catálogo de los TT.00. TTO0, en particular la inserción en catálogos.

Garantizar los planes de Grupos FAM. 
Aumentar la participación en Ferias.

$\begin{array}{llll}\text { 12. Presencia } & \text { Implementar } & \text { un Diseñar mecanismos en la plataforma } \\ \text { en principales } & \text { Plan } & \text { de Internet } & \text { para } \\ \text { buscadores de } & \text { Comunicación } & \text { promocionales y de ventas. } & \\ \text { Internet. } & \text { Integral para } & & \\ & \text { promover el sitio } & \\ & \text { de ventas del } & \end{array}$

13. Presencia Incluirse en el GDS Negociar con el GDS Amadeus los términos en GDS.

Amadeus. del contrato.

14. Ventas on- Implantar otras Actualizar tecnológicamente las soluciones line soluciones

del del sistema informático del Grupo hacia las Sistema de Gestión facilidades de Internet. Zun.

Emplear el sitio web como herramienta para el mayor posicionamiento en las ventas de los hoteles y la actualización de información sobre los productos-servicios.

15. Sistemas de Implantar nuevas Optimizar la conectividad y las redes para facilidades en los informatización e incremento de las ventas Sistemas de on line.

\section{gestión Gestión Comercial \\ comercial (software).}

16. Programas Mantener el Ampliar los horizontes de los programas a de fidelización control y mejora nuevos nichos de mercados que garanticen al cliente continua de los una mayor diferenciación de los productosPlanes de servicios, y no solamente a los segmentos Fidelización para principales. las marcas hoteleras.

\section{Conclusiones}

A partir de la aplicación del análisis multicriterio para determinar los factores claves del éxito que inciden en la situación competitiva de los productos-servicios hoteleros de ciudad del Grupo Gaviota S.A, y en consecuencia realizar una propuesta de estrategias para la empresa se puede arribar a las siguientes conclusiones:

- Del análisis teórico de la competitividad empresarial se obtiene la interrelación entre Ventajas Competitivas a alcanzar por la organización, FCE a dominar en el sector y Capacidades Distintivas indispensables a desarrollar desde su gestión en los hoteles de ciudad del Grupo de Turismo Gaviota S.A.

- Para los hoteles de ciudad en el destino La Habana se identificaron 16 factores claves de éxito que inciden en la satisfacción de los clientes con respecto al 
producto-servicio, así como la posición ventajosa del Grupo Gaviota S.A con respecto a los Grupos Cubanacán y Gran Caribe en más del $85 \%$ de estos.

- El sector hotelero de "Ciudad" de La Habana, Cuba; se caracteriza por una elevada rivalidad competitiva entre las empresas, por lo que Gaviota para mantenerse y mejorar en dicho escenario, debe modificar el producto-servicio y aumentar su política comercial.

- El Grupo Gaviota no disfruta de ventajas competitivas en las 5 áreas claves para analizar los factores claves de éxito, pero los domina en un grado superior al de sus principales competidores entre los grupos de turismo cubanos.

- El estudio realizado permitió establecer la relación entre los FCE, las Capacidades Distintivas necesarias a desarrollar por el grupo para elevar su competitividad en el segmento hotelero de ciudad con las posibles estrategias y fuentes de ventajas para la organización.

\section{Referencias bibliográficas}

Eccles, P. Planning for Improved Performance. En: Management Accounting. pp 53-54. 1993.

Gárciga, R.J. "Formulación Estratégica, un enfoque para directivos”. Editorial Félix Varela, La Habana. 1999.

Johnson, G. y Acholes, K. Exploring corporate strategy. Prentice Hall, Hertfordshire. 2002 .

Porter, M. How information gives you competitive advantage". Harvard Business Review. Vol 63, 149-150. (1985).

Porter, M "Ventaja competitiva. Creación y sostenimiento de un desempeño superior". Editorial CECSA. México (1990).

Porter, M Ventaja Competitiva, CECSA, México. (1988).

Ronda, G.A. Dirección estratégica, constructo y dimensiones. Ediciones Futuro. La Habana. 2015.

Ronda, G.A. Dirección Estratégica integrada: Conceptualización en las condiciones de Cuba.Editorial Felix Varela. 2016.

Wootton, S. y Horne, F. Pensamiento Estratégico: un camino a la estrategia paso a paso. Ediciones Giga. 2012.

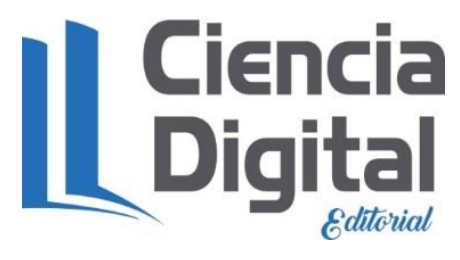




\section{PARA CITAR EL ARTÍCULO INDEXADO.}

Spengler Betancourt, M. E., \& Ascón Villa, J. E. (2021). Estrategia de mejora para el aumento de la competitividad de los productos - servicios hoteleros de ciudad del grupo de turismo Gaviota s.a. Explorador Digital, 5(1), 350-364. https://doi.org/10.33262/exploradordigital.v5i1.1507

\section{Ciencia \\ LDigital}

El artículo que se publica es de exclusiva responsabilidad de los autores y no necesariamente reflejan el pensamiento de la Revista Explorador Digital.

El artículo queda en propiedad de la revista y, por tanto, su publicación parcial y/o total en otro medio tiene que ser autorizado por el director de la Revista Explorador Digital.
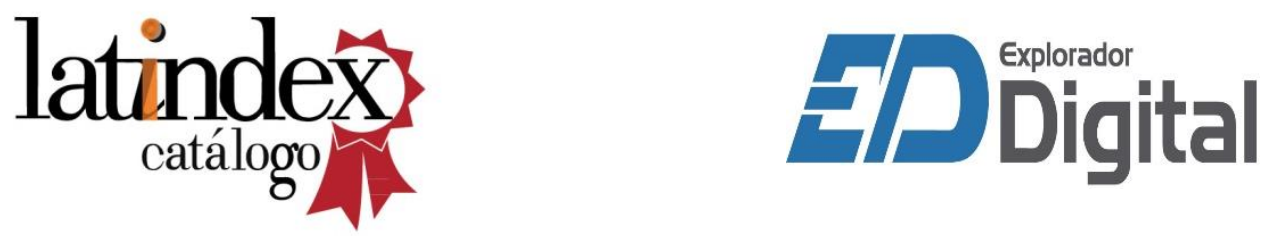